\title{
Endoscopic Removal of Inflated Transected Sengstaken-Blakemore Tube Using Endoscopic Scissors
}

\author{
Jun Ho Lee ${ }^{1}$, Eu-Kwon Hwang ${ }^{1}$, Chanmesa Doeun², Jeong-Ju Yoo', Sang Gyune Kim¹ and Young Seok Kim ${ }^{1}$ \\ ${ }^{1}$ Digestive Disease Center and Research Institute, Department of Internal Medicine, Soonchunhyang University College of Medicine, \\ Bucheon, Korea, ${ }^{2}$ Department of Internal Medicine, Battambang Referral Hospital, Battambang, Cambodia
}

Balloon tamponade using Sengstaken-Blakemore (SB) tube is employed as a bridging therapy in cases in which endoscopic therapy fails to control esophageal variceal bleeding. Although SB tube insertion can lead to successful hemostasis, it is accompanied by numerous complications, with SB tube transection being one of the rarest complications. A 53-year-old man with liver cirrhosis and hepatocellular carcinoma presented with massive esophageal variceal bleeding. Therapeutic endoscopic variceal ligation failed, and SB tube was inserted. The SB tube was unexpectedly disconnected because of the patient's irritability due to hepatic encephalopathy. The esophageal and gastric balloon of the SB tube remained inflated in the stomach. Whereas the use of other endoscopic instruments was ineffective, endoscopic removal was successfully accomplished using endoscopic scissors. In conclusion, we detected SB tube transection in a patient with hepatic encephalopathy and removed remnants of the inflated tube using endoscopic scissors. Clin Endosc 2019;52:182-185

Key Words: Sengstaken-Blakemore tube; Transection; Impaction; Endoscopic scissors

\section{INTRODUCTION}

Balloon tamponade is often used as a bridging therapy when immediate endoscopy or endoscopic control of variceal bleeding is difficult. ${ }^{1}$ It is known to be particularly effective in treating massive variceal bleeding and is recommended by clinical guidelines. ${ }^{2}$ Sengstaken-Blakemore (SB) tube is the most widely used instrument for balloon tamponade. ${ }^{3}$ Inflation of the esophageal and gastric balloon of the SB tube and anchoring it against the gastroesophageal junction are critical for adequate tamponade when using the SB tube. Appropriate

Received: May 1, 2018 Revised: June 15, 2018

Accepted: June 21, 2018

Correspondence: Young Seok Kim

Digestive Disease Center and Research Institute, Department of Internal Medicine, Soonchunhyang University College of Medicine, 170 Jomaru-ro, Wonmi-gu, Bucheon 14584, Korea

Tel: +82-32-621-5079, Fax: +82-32-621-5072, E-mail: dr.yskim@gmail.com ORCID: https://orcid.org/0000-0002-7113-3623

(cc) This is an Open Access article distributed under the terms of the Creative Commons Attribution Non-Commercial License (http://creativecommons.org/ licenses/by-nc/3.0) which permits unrestricted non-commercial use, distribution, and reproduction in any medium, provided the original work is properly cited.
SB tube placement results in successful hemostasis $(40 \%-$ $90 \%) .{ }^{4}$ However, it is associated with several major complications, including esophageal rupture, aspiration pneumonia, esophageal ulcers, and asphyxia resulting from balloon migration. ${ }^{3}$ Impaction of the SB tube is a very rare complication, and endoscopic removal of an impacted SB tube has been reported in only 5 cases worldwide. In real clinical practice, impairment in liver function typically observed in patients requiring SB tube insertion is accompanied by hepatic encephalopathy. In grade 1 and 2 hepatic encephalopathy, the possibility of irritability in patients is increased, and the SB tube is transected and impacted. According to previous reports, most impacted SB tubes were deflated and easily removed endoscopically. However, under conditions when the SB tube is transected and the remnant tube remains inflated, endoscopic removal of the remnant tube is unknown endoscopically, and the choice of endoscopic tool is not established in this rare situation.

Here, we reported an exceedingly rare case of endoscopic removal of transected SB tube with inflated esophageal and gastric balloon. 


\section{CASE REPORT}

A 53-year-old man with no history of specific disease presented with abdominal pain. He visited another hospital and underwent laboratory tests and abdominal computed tomography. He was diagnosed with chronic hepatitis B and liver cirrhosis (LC) associated with hepatocellular carcinoma (HCC) and was referred to our hospital for further work-up and suitable treatment.

At the time of admission, the patient's blood pressure and pulse rate were 140/90 $\mathrm{mm} \mathrm{Hg}$ and 90 beats per minute, respectively. Further, his breathing rate and body temperature were 22 breaths per minute and $36.4^{\circ} \mathrm{C}$, respectively. Abdominal distension and shifting dullness were observed, whereas no palpable mass, tenderness, or rebound tenderness was detected in the abdomen. The results of complete blood cell count were as follows: white blood cell count, $5,630 / \mathrm{mm}^{3}$; hemoglobin, $9.7 \mathrm{~g} / \mathrm{dL}$; hematocrit, $28.5 \%$; and blood platelet count, $73,000 / \mathrm{mm}^{3}$. Moreover, the results of serum biochemical test were as follows: total protein, $6.0 \mathrm{~g} / \mathrm{dL}$; albumin, 1.8 $\mathrm{g} / \mathrm{dL}$; total bilirubin, $1.53 \mathrm{mg} / \mathrm{dL}$; aspartate aminotransferase, $95 \mathrm{IU} / \mathrm{L}$; alanine aminotransferase, 15 IU/L; alkaline phosphatase, 697 IU/L; gamma-glutamyltranspeptidase, $166 \mathrm{IU} / \mathrm{L}$; blood urea nitrogen, $19.4 \mathrm{mg} / \mathrm{dL}$; and creatinine, $1.0 \mathrm{mg} / \mathrm{dL}$. Prothrombin time and activated partial thromboplastin time were within normal limits.

Abdominal ultrasonography, dynamic magnetic resonance imaging, and esophagogastroduodenoscopy (EGD) were performed, and ultimately, the patient was diagnosed with LC, HCC infiltrating both the liver lobes with portal vein thrombosis, grade 2 esophageal varices with red color sign, and type 2 gastroesophageal varices (GOV2). On day 4 of hospitalization, he underwent prophylactic endoscopic variceal ligation (EVL) and transarterial chemoembolization in accordance with the Barcelona Clinic Liver Cancer guidelines for HCC. Despite appropriate management, the patient's liver function gradually deteriorated, and esophageal variceal bleeding (EVB) and hepatic encephalopathy recurred during admission.

On day 12 of hospitalization, he presented with hematemesis (400 $\mathrm{mL}$ of blood) resulting from EVB and was immediately transferred to the intensive care unit. EGD and therapeutic EVL, along with aggressive blood volume resuscitation and intravenous administration of terlipressin, were performed to control EVB but failed because of unstable vital signs and massive bleeding. SB tube insertion (All Silicone SengstakenBlakemore Tube ${ }^{\circledR}$; Create Medic Co., Yokohama, Japan) was performed as subsequent treatment, and medical therapy including administration of terlipressin was continued. On day 13 of hospitalization, grade 2 hepatic encephalopathy developed, and the SB tube was abruptly cut off because of the patient's irritability.

Emergency EGD showed esophageal and gastric balloon transection from the SB tube, which remained fully inflated and impacted. We attempted to deflate the balloon using various methods based on previous reports. Endoscopic injection needle (WS-2416PN2504; Omnimed Ltd., England, UK) and endoscopic cutter (FS-5L-1; Olympus, Tokyo, Japan) were initially used, but the balloon, having thick elastic walls, was not easily punctured. We subsequently used endoscopic hot biopsy forceps (FD-1U-1; Olympus) and employed argon plasma coagulation, which proved unsuccessful as the balloon, which was made of silicone, had excellent electrical insulating and heat-resistant properties. On the presumption that a sharper and more rigid instrument could cut the balloon, we finally chose and used endoscopic scissors (FS-3L-1; Olympus) to perforate the balloon (Fig. 1), and the deflated balloon was gently removed using an endoscopic snare (SD-210U-10; Olympus) without any complication (Fig. 2).

\section{DISCUSSION}

EVB is a lethal complication of portal hypertension in patients with $\mathrm{LC}^{5}$, with a mortality rate ranging from $12 \%$ to $20 \%$ during the acute episode, which, by consensus, refers to the first 5 days after patient's hospital visit. ${ }^{3}$ Over the past few decades, numerous treatment options for the management of EVB have been used, such as pharmacological vasoconstriction, physical compression via balloon tamponade, and diverse endoscopic interventions. ${ }^{5}$ The recommended treatment for acute EVB is based on early and circumspect blood volume resuscitation and early administration of intravenous vasoactive drugs (terlipressin, somatostatin, or analogs) and prophylactic antibiotics. Within the first 6 to $12 \mathrm{~h}$ of admission following hemodynamic stabilization, EGD was used to confirm the origin of bleeding and perform endoscopic therapy. ${ }^{3} \mathrm{EVL}$ is the preferred endoscopic therapy for EVB, whereas endoscopic variceal obturation is recommended for acute bleeding resulting from GOV2 and isolated gastric varices. ${ }^{6}$ The optimal endoscopic therapy for type 1 gastroesophageal varices remains controversial. ${ }^{7}$

However, the recommended treatment fails to control bleeding in $10 \%-20 \%$ of cases. ${ }^{8}$ If standard therapy fails to control EVB or patients are hemodynamically unstable to undergo endoscopic procedure, balloon tamponade using an SB tube is the next treatment option. ${ }^{1,2}$ Although other endoscopic procedures such as self-expanding metal stenting can be employed, SB tube insertion remains to have a role to play in the management of EVB. ${ }^{2}$ Nevertheless, SB tube insertion is accompanied by various complications, some of which are 
major or minor events. ${ }^{9}$ In our case, definitive airway protection was provided before SB tube placement to prevent various complications. The gastric balloon was placed in the stomach fundus and inflated with $250 \mathrm{~mL}$ of air, whereas the esophageal balloon was inflated to a pressure of $30 \mathrm{~mm} \mathrm{Hg}$ and deflated every $6 \mathrm{~h}$.

Among various complications, impaction of the SB tube is exceptionally rare, and endoscopic removal of the impacted SB tube has been reported in only 5 cases worldwide (Table 1). In 1985, Gossat and Bolin ${ }^{10}$ reported a case of endoscopic removal of inflated esophageal and gastric balloon transected from the SB tube using endoscopic biopsy forceps. In contrast, Chawla et al. ${ }^{11}$ in 1988 and Kim et al. ${ }^{12}$ in 2006 reported a case of endoscopic removal of inflated gastric balloon not transected from the SB tube using endoscopic sclerotherapy needle and using endoscopic needle knife and sclerotherapy needle, respectively, whereas Ko et al. ${ }^{4}$ in 2010 reported a case of endoscopic removal of inflated gastric balloon transected from the SB tube using endoscopic injection needle. Moreover, in 2013, Gonçalves et al. ${ }^{13}$ described a case of endoscopic removal of deflated esophageal and gastric balloon transected from the SB tube using endoscopic snare. As in previously reported cases, forcefully pulling the inflated balloon of the SB tube must be avoided to prevent lethal complications such as hemorrhage, perforation, and airway obstruction. ${ }^{4,12}$

SB tube was transected in our case, unlike in the cases
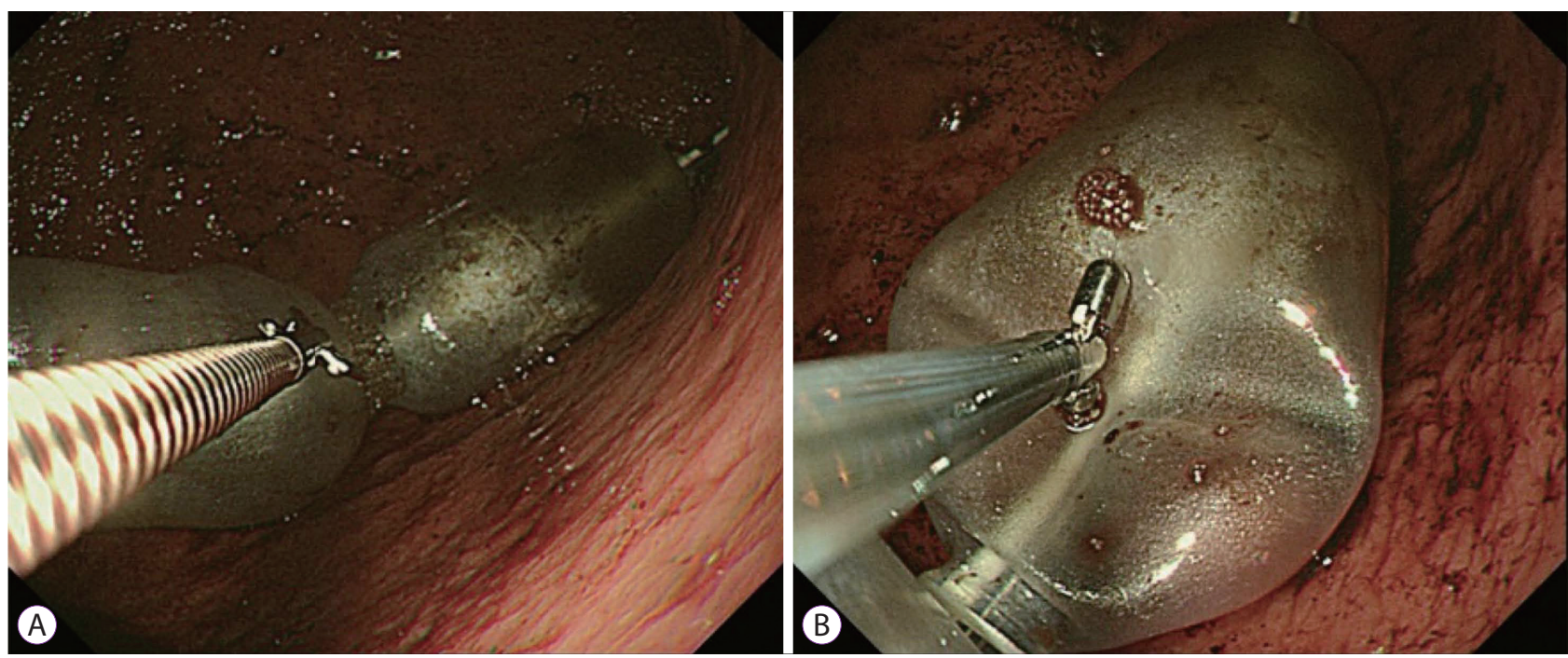

Fig. 1. (A, B) Deflation of impacted esophageal and gastric balloon transected from the Sengstaken-Blakemore tube using endoscopic scissors.
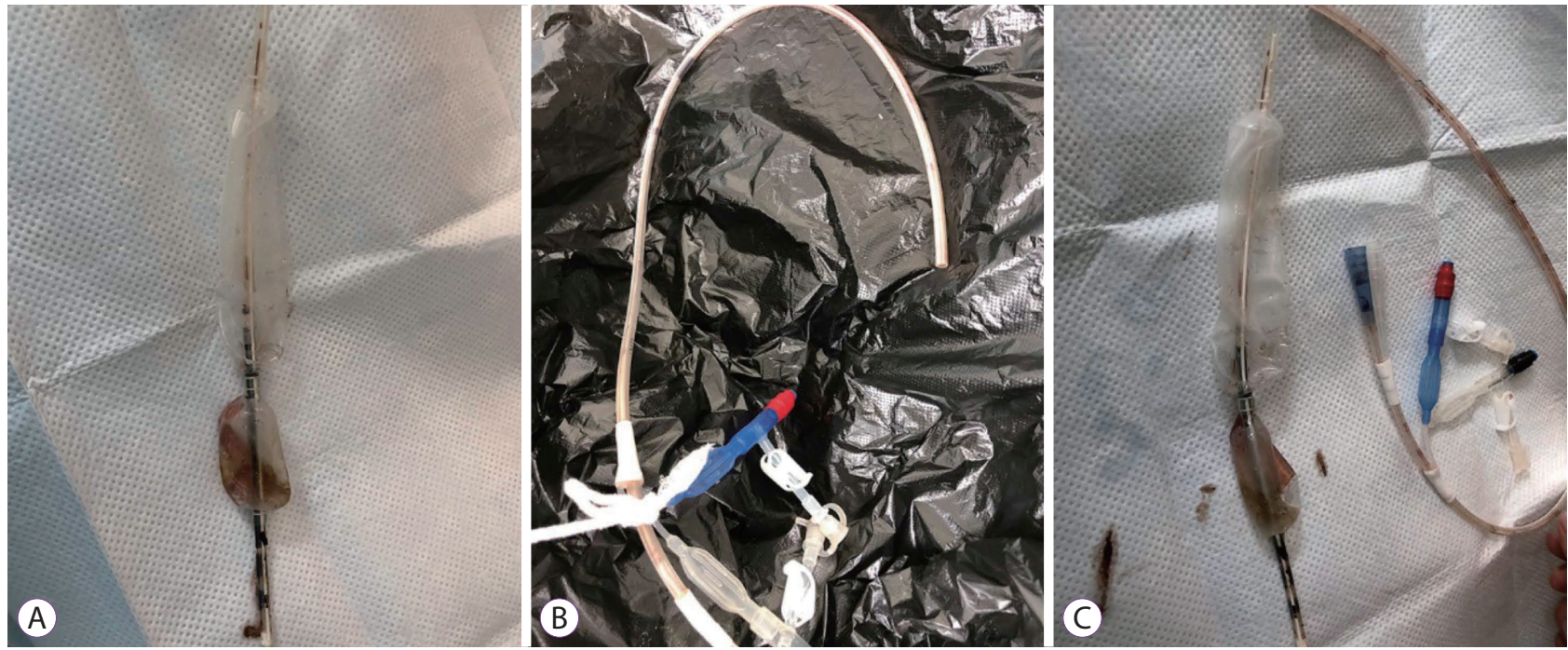

Fig. 2. (A) Deflated esophageal and gastric balloon extracted outside. (B) Transected remnant tube excluding esophageal and gastric balloon. (C) Entire transection of the Sengstaken-Blakemore tube. 
Table 1. Literature Review of Endoscopic Removal of Impacted Sengstaken-Blakemore Tube

\begin{tabular}{llccl}
\hline Author & \multicolumn{1}{c}{ Impacted balloon type } & Ballooning & Transection & \multicolumn{1}{c}{ Endoscopic tool used } \\
\hline Gossat et al. $^{10}$ & Esophageal and gastric balloon & Inflated & Transected & Endoscopic biopsy forceps \\
Chawla et al. $^{11}$ & Gastric balloon & Inflated & None & Endoscopic sclerotherapy needle \\
Kim et al. $^{12}$ & Gastric balloon & Inflated & None & Endoscopic needle knife and sclerotherapy needle \\
Ko et al. $^{4}$ & Gastric balloon & Inflated & Transected & Endoscopic injection needle and snare \\
Gonçalves et al. $^{13}$ & Esophageal and gastric balloon & Deflated & Transected & Endoscopic snare \\
\hline
\end{tabular}

reported by Ko et al. ${ }^{4}$ and Gonçalves et al., ${ }^{13}$ because of the patient's irritable movement rather than SB tube deformation resulting from inappropriate sterilization and reuse or from manufacturing defect. Being a new product, the SB tube was carefully inspected prior to intubation. To prevent various complications associated with SB tube insertion, it is important not only to fully understand the procedure and inspect the SB tube before insertion but also to closely monitor the patient after intubation. ${ }^{4}$

We attempted to puncture the inflated gastric and esophageal balloon transected from the SB tube using endoscopic injection needle, as reported by Ko et al., ${ }^{4}$ and endoscopic hot biopsy forceps and argon plasma coagulation, similar to Kim et al., ${ }^{12}$ but to no avail. In our case, because of the excellent electrical insulating and heat-resistant properties of the SB tube, which was made of silicone, the use of endoscopic hot biopsy forceps and argon plasma coagulation might not have worked. Various factors including endoscopists' technique, sort of instrument such as endoscopic injection needle type, and balloon characteristics may have an effect on the success of balloon puncture. In our case, we presumed that a sharper and more rigid instrument might work and finally used endoscopic scissors to puncture the balloon's thick and elastic walls.

To the best of our knowledge, no previous report has described the endoscopic removal of impacted esophageal and gastric balloon transected from the SB tube, which is extremely rare, using endoscopic scissors. Here, we reported a very rare case of endoscopic removal of inflated transected SB tube using endoscopic scissors. After intubation, close patient monitoring is crucial, and appropriate sedation can be used to prevent SB tube transection owing to the patient's irritable movement. ${ }^{4}$ If the SB tube is transected and its esophageal and gastric balloon remains inflated, leading to impaction, endoscopic removal using endoscopic scissors can be considered an effective treatment option.

Conflicts of Interest

The authors have no financial conflicts of interest.
Acknowledgements

This work was supported by the Soonchunhyang University Research Fund.

\section{REFERENCES}

1. Kim YD. Management of acute variceal bleeding. Clin Endosc 2014;47:308-314.

2. Garcia-Tsao G, Abraldes JG, Berzigotti A, Bosch J. Portal hypertensive bleeding in cirrhosis: risk stratification, diagnosis, and management: 2016 practice guidance by the American Association for the study of liver diseases. Hepatology 2017;65:310-335.

3. Escorsell À, Pavel O, Cárdenas A, et al. Esophageal balloon tamponade versus esophageal stent in controlling acute refractory variceal bleeding: a multicenter randomized, controlled trial. Hepatology 2016;63:19571967.

4. Ko DH, Kim CW, Lim CH, et al. A case of endoscopic removal of a broken off gastric balloon from a Sengstaken-Blakemore tube. Korean J Gastrointest Endosc 2010;40:249-251.

5. Dechêne A, El Fouly AH, Bechmann LP, et al. Acute management of refractory variceal bleeding in liver cirrhosis by self-expanding metal stents. Digestion 2012;85:185-191.

6. Kim SJ, Choi CW, Kang DH, et al. Emergency endoscopic variceal ligation in cirrhotic patients with blood clots in the stomach but no active bleeding or stigmata increases the risk of rebleeding. Clin Mol Hepatol 2016;22:466-476.

7. Park SJ, Kim YK, Seo YS, et al. Cyanoacrylate injection versus band ligation for bleeding from cardiac varices along the lesser curvature of the stomach. Clin Mol Hepatol 2016;22:487-494.

8. Garcia-Tsao G, Bosch J. Management of varices and variceal hemorrhage in cirrhosis. N Engl J Med 2010;362:823-832.

9. Bhasin DK, Zargar SA, Mandal M, Goenka M, Singh R. Endoscopic removal of impacted Sengstaken-Blakemore tube. Surg Endosc 1989;3:5455.

10. Gossat D, Bolin TD. An unusual complication of balloon tamponade in the treatment of esophageal varices: a case report and brief review of the literature. Am J Gastroenterol 1985;80:600-601.

11. Chawla Y, Singh R, Ramesh GN, Dilawari JB. Impacted Sengstaken Blakemore tube. Am J Gastroenterol 1988;83:1438-1439.

12. Kim IH, Koh KH, Kim SH, Kim SW, Lee SO, Lee ST. A case of endoscopic removal of impacted Sengstaken-Blakemore tube. Korean J Gastrointest Endosc 2006;32:271-274.

13. Gonçalves BM, Caetano AC, Fernandes D, Cruz A, Bastos P, Rolanda C. Sengstaken-Blakemore tube: an unusual complication. Endoscopy 2013;45 Suppl 2 UCTN:E434. 